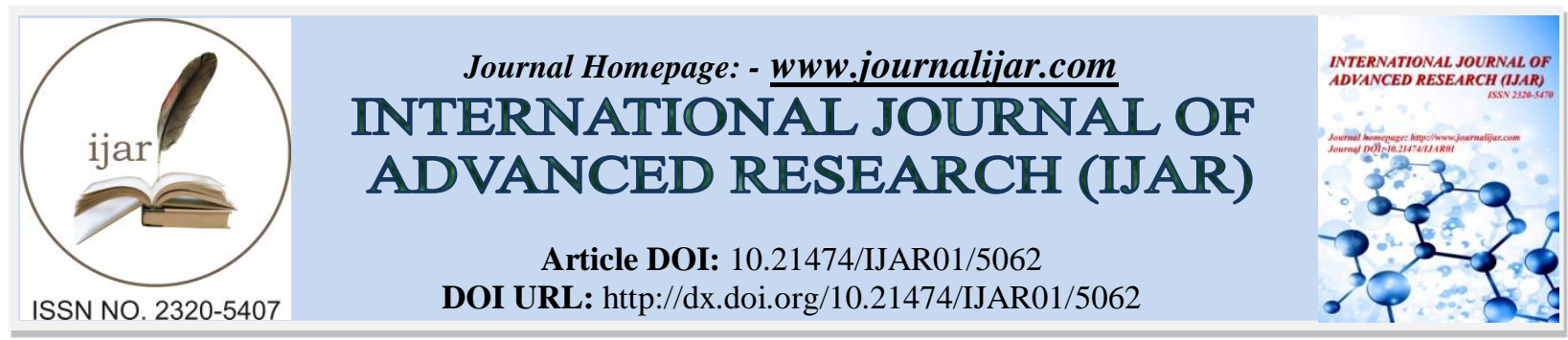

RESEARCH ARTICLE

\title{
ASSESSMENT OF CURRENT STATUS OF NURSING PRACTICES AT KABUL BASED HOSPITALS.
}

Rafiullah Safi.

Jodhpur School of Public Health.

\section{Manuscript Info}

(.........................

Manuscript History

Received: 04 June 2017

Final Accepted: 06 July 2017

Published: August 2017

Key words:-

Standard precautions, Precautions, Standard, precautions practices.

\section{Abstract}

Hospital infections, which pose a serious problem, and can threat the health and safety of patients and medical staff worldwide. These infections from one side affect the quality of health care service delivery from other side it increases cost of medical care in the hospitals. In order to avoid this situation, it becomes highly important to delivery health care and nursing care based on some specific and context oriented standards, which if followed in the hospitals, can protect patients as well as medical workers and help to control the occurrence of hospital acquired infections.

To explore nurses' understanding about standard precaution measures in the target hospitals.

To find out what challenges are associated with implementing the universal standard nursing practices.

To provide valid recommendations for improving current practices.

The methods used in research study, contains both qualitative and quantitative methods, and both of them are complementing each other. The research was completed using a comprehensive questionnaire. The questionnaire was filled through conducting structured and semi structured interviews, focus group discussions, and the data was triangulated by observations. The research study was conducted in governmental, semi- governmental and private hospitals.

1. The average nurse bed ratio in all governmental, PPPs and private hospitals was found to be 0.5 , which is normal based on the WHO standards.

2. The number of the female nurses are very low compared to male nurses.

3. Most of the nursing department heads have received various training from MOPH and only 15\% of nursing heads did not receive any nursing care training.

4. $82 \%$ of the hospitals had not received standard guidelines from the $\mathrm{MOPH}$, and only $18 \%$ of the hospital received standard written nursing guidelines.

5. $81 \%$ of the nurses interviewed didn't have knowledge on nursing standard and precaution methods and procedures.

6. $76 \%$ of health facilities were monitored by the MOPH and $24 \%$ of the hospital were not monitored by MOPH.

Regular training on full package of nursing guidelines should be provided to the nursing staff to build their capacity. 
Required infection prevention materials and medicine for emergency patient should be given in enough quantity and on timely manner to the nursing care departments.

Access to the nursing guideline should be given to all hospitals through uploading in the MOPH website.

Monitoring and feedback mechanism should be in place for nursing practices and nursing care should be monitored on regular basis from the MOPH.

Exchange learning programs from government to PPPs and peer learning should be introduced to build the capacity of the government hospital nursing staff.

Copy Right, IJAR, 2017,. All rights reserved.

\section{Introduction:-}

Hospital infections, which pose a serious problem, and can threat the health and safety of patients and medical staff worldwide. These infections from one side affect the quality of health care service delivery from other side it increases cost of medical care in the hospitals. In order to avoid this situation, it becomes highly important to delivery health care and nursing care based on some specific and context oriented standards, which if followed in the hospitals, can protect patients as well as medical workers and help to control the occurrence of hospital acquired infections. It is mentionable that MOPH has also prepared standard nursing guidelines, in 2013 and a copy of these guidelines have been distributed in most of the governmental hospitals supervised by MOPH. The purpose of distribution to the guideline was to be followed by nursing staff during their daily practices and protect them from harming of occupational hazards.

This research paper mainly focused on only few dimensions that were found to be affecting nursing standards of health care service delivery. These elements were explored during the initial assessment and reviewing of National Policy and Strategy for Nursing and Midwifery Services 2011 - 2015, and conducting direct interviews with nursing staff of Kabul based hospitals, prior to the original research, which included but not limited to;

Current hospital nursing practices

Availability of written nursing guidelines

Awareness level of respondents on Nursing standards Nurse bed ratio (

Average required Nursing Staff)

Qualification of nursing staff Gender

equality

Capacity building of the nursing staff or short term trainings Conducted to the Nursing staff Monitoring mechanism of nursing standards

However; the National Policy and Strategy for Nursing and Midwifery Services 2011 - 2015, prepared by the MOPH also focused on the Mentioned points. In addition to that various data collection tools were used such as (questionnaire, focus group discussions, structure interviews and semi structure interviews), and the collected data was verified by using triangulation before finalizing the research data.

\section{Problem Statement:-}

Kabul, as one of the overpopulated province and capital city in Afghanistan. The government here in Kabul make the policies for reform and development and then its rollout throughout the whole system in Afghanistan, which is really challenging. In addition to that health services are currently provided by three type of hospitals: Public, Public-Private and Private.

Experiencing timely receiving of the health services, it is observed that nurses in most of the mentioned three types of health facilities, caring the patients are actually not considering and following standard precautions.

In a recent prevalence survey from the WHO shows that $8.7 \%$ of in-patients acquire infections in hospitals. In addition to that the report also says that the incidence rate of needle stick injury in nurses is $80.6 \%$ in the hospital. This means that the nurses who are caring the patients in health facilities are highly prone to various type of serious contagious diseases if they don't follow the standard precaution methods. 
Hence it becomes highly important to explore on current status of nursing practices at Kabul based hospitals, and provide them valid recommendations to improve their occupational health and safety. (WHO, 2002)

This makes both the nurse and patient prone to various health problems. Especially infectious and blood borne diseases. Health care workers (HCWs) are at risk of occupational hazards because they perform their clinical activities in hospitals. They are exposed to blood-borne infections from pathogens such as human immunodeficiency virus (HIV), hepatitis B virus (HBV) and hepatitis C virus (HCV), and from sharps injuries and contact with deep body fluids.

HCWs working in hospitals frequently provide care to patients whose HIV, HBV, and HCV statuses are unknown.

Thus the researcher intended to conduct a research study and explore on currently nursing practices in Afghanistan and ensure that, whether the nursing staff have awareness on nursing care standards, and whether if they are currently practicing as per the nursing guidelines. As well as explore further, if the standards are not being followed. Furthermore; the research also provides concrete suggestions for improvement of the currently used practices by the nurses in the target hospitals.

\section{Research Variables:-}

The research is designed to be purely a qualitative study, therefore the variables that are followed into the research are also qualitative one. Among the qualitative variables mostly ordinal qualitative variables were used.

Following are the variables used to obtain certain information for the research study in the target hospitals:

\section{Dependent variables:}

Current hospital nursing standards or practices of the hospital

\section{Independent variables:}

Age, Sex,

Knowledge, Public

Private

Availability of written nursing guidelines

Overall awareness level of respondents on Nursing standards Nurse bed ratio ( Average required Nursing Staff) Qualification of nursing staff Gender equality

Capacity building of the nursing staff or short term trainings Conducted to the Nursing staff Working experience Monitoring mechanism of nursing standards

\section{Rational:-}

As it is clear that hospital is a place where health workers are to interact with all the types of contagious and noncontagious diseases. Therefore; chance of disease transmission from one person to another person increases. In order to save health worker from transmitting the infections during the nursing processes, it is required to apply a set of planned actions and measure that protect nurses, doctor and patients from diseases. Secondly it is essential to protection department of the hospital, to control the transmission of the diseases, and this objective can be only achieved when standard precaution methods are followed for all OPD and IPD patients while caring patients by nursing personnel.

Moreover; the research focused on essential thematic areas for the purpose to improve the quality of nursing services delivery in the target hospitals.

This research has also provided reference evidence for the relevant departments, which can assist them in formulating policies, to prevent nosocomial infections. Furthermore; the research proposes specific trainings to the nurses, which will be adding greater value to the protection procedures and reducing the harm cycle from nurse to the patients and vice-versa. 


\section{Study Objectives:-}

Following are the research objectives that were considered throughout the study in the targeted hospitals of Kabul province:

To explore nurses' awareness level about standard nursing practices in the target hospitals.

To find out what challenges are associated with implementing the universal standard nursing practices.

To provide valid recommendations for improving current practices.

\section{Research Question:-}

Considering the current nursing practices in Kabul based hospital, it is observed and found during the interviews, that most of the nurses are not following the standard guidelines for nursing care in delivering of nursing services to the patients. Thus this has caused the transmissions of various infectious diseases to both the patients and nurses. This fact is not only the visible reality in Afghanistan, but this is also observed in a prevalence survey conducted by the WHO in other countries as well. Thus the researcher conducted study on to explore the current level of the nursing standards, the reasons why the standard nursing practices are not followed, and the how to improve the current nursing care in the hospitals. Below is the research question for the study conducted throughout the 26 targeted hospitals.

\section{What is the Current status of nursing practices at Kabul Based Hospitals? Importance of the research to the Priority area of the country:-}

The current health services that are being delivered by the Afghan professional seems to be not meeting the universal health standards, and it was expressed in one of the research conducted by Dr.Niash. and evidence to support this statement is, that most of the Afghan national visit Pakistan or India if they get any serious diseases. Therefore; referencing this study, it is highly required to focus more on the meeting the standards for reatment rather than only delivering health services. And if we think of retaining Afghans patients to get treated in country, we need to make improvement in the current health services system. And make our level of health services delivery equal to the neighboring countries. This should include standardizing not only in the nursing care, but should also include the all other sections of health services delivering health services in the country.

\section{Opportunity and Challenges:-}

This research was actually challenging for so many reasons. Initially the literature review for this research was not available for Afghanistan, this means this was not previous study or data available to explore information on nursing practices in Kabul based hospitals. Secondly, the research was looking a fter the strong and weak points for nursing practices, therefore; the hospital managers in most of the time were not cooperative with the researcher.

The opportunity was that MOPH helped me with introduction letters to all the relevant hospitals, and the researcher had the knowledge and good experience of conducting such a larger researches in the past.

\section{Literature Review:-}

Health care workers (HCWs) are at risk of occupational hazards because they perform their clinical activities in hospitals. They are exposed to blood-borne infections from pathogens such as human immunodeficiency virus (HIV), hepatitis B virus (HBV) and hepatitis C virus (HCV), and from sharps injuries and contact with deep body fluids.

HCWs working in hospitals frequently provide care to patients whose HIV, HBV, and HCV statuses are unknown. Studies have shown that there is a $0.3 \%$ - $0.5 \%$ occupational risk of infection with HIV after percutaneous exposure to HIV-contaminated blood, and the cumulative career risk may be as high as 1\%-2\% among emergency service staff or surgeons. A survey assessing exposure to HIV among HCWs in South Africa showed that $13.0 \%$ of the staff reported accidental exposure when caring for HIV positive patients. A study in Sweden showed that the majority of reported cases of occupational blood exposure were among nurses.

Another study showed that nurses were the staff most frequently involved in occupationally acquired HIV infection. A study assessing the frequency of body fluid exposure among midwives showed that $65.1 \%$ of them had experienced exposure to amniotic fluids or blood at least once in the past six months and that $25.0 \%$ reported five or more times that level of exposure.

In developing countries, a lack of resources and a lack of appropriate legislation and control are the main reasons 
for the inappropriate management of health care waste. HCWs' low awareness of the required standards can make the situation worse. However, with limited resources, organizations can put forward appropriate rules and control systems, and raise HCWs' awareness of international standard practices of healthcare waste disposal. In Afghanistan, little information is available about the prevalence of HIV, hepatitis B surface antigen (HBsAg), and $\mathrm{HCV}$ among the overall population. However, Todd et al. reported that among injecting drug users (IDUs) in Kabul, the prevalence of HBsAg-positive, HCV-positive, and HIV-positive patients was 6.5\%, 36.6\%, and 3.0\%, respectively. Universal precautions (UPs) are a set of precautions or actions designed to prevent HCWs from being exposed to blood and deep body fluids by applying the basic principles of infection control through hand washing, utilization of appropriate protective barriers such as gloves, masks, gowns, and eye shields, safe handling and disposal of needles, and safe decontamination of instruments and other contaminated equipment. The term standard precautions are replacing universal precautions, as it expands the coverage of universal precautions by recognizing that anybody fluid may contain contagious and harmful microorganisms.

The level of practice of universal precautions by HCWs may differ from one type of HCW to another.

The differences in knowledge of universal precautions by HCWs may be influenced by several factors that can lead to poor compliance with universal precautions. The absence of an enabling environment in the health institution, such as a lack of constant running water or a shortage of personal protective equipment (PPE), can lead to poor compliance with universal precautions. UPs are important because any health care organization has a responsibility to protect its staff from potential dangers and itself from loss of manpower due to occupational injuries or illnesses. Patients may be harmed if staff are uninformed about safe handling of blood or body fluids, and they may be deprived of appropriate care due to HCWs' inappropriate fears or misunderstandings.

Surveys have shown that the use of UPs significantly decreases the number of incidents of occupational exposure to blood. Nevertheless, the level of compliance with UPs has been reported to be generally lo. The reported weakest aspects were not practicing hand decontamination, not using barrier protection, and not recapping needles. A study of occupational injury history and awareness of UPs showed a low level of knowledge of the basic principles of UPs among HCWs in hospitals in Kabul. Although a few studies have been conducted regarding knowledge of UPs among HCWs in Afghanistan, there has been no study of practice. This cross-sectional study aimed to assess HCWs' knowledge and practice of UPs and to examine the associations between their knowledge and practice.

Viruses, other micro-organisms and chemicals that could have caused harm or diseases from hands (Suzzame et al., 2008). The attitude of some health workers towards hand washing, if improved can help reduce the transmission of micro-organisms. According to Sobayo (2005), patient may develop urinary tract infection due to improper care of urethral catheter, wound infection due to poor operative technique, use of improperly sterilized instrument and inadequate care of wound, phlebitis and septicaemia due to improper handling of intravenous infusion administration. All these are unnecessary infections and lead to extra cost to hospital and the patients.

Failure to comply with policies and procedures that support the reduction of hospital acquired infections (HAIs) is a recognized and complex problem that may be contributing to the current trend in the world (Damina, 2006). Research has identified varying degree of noncompliance with standard precaution. According to Centre for Disease Control (2002), limited knowledge, lack of facilities and poor working environment are commonly cited as barriers to compliance. WHO (2004) opined that the risk of professional exposure to viruses is compounded by restricted size of staff in many health units, the lack of basic protective equipment, cleaning materials and deficit knowledge contributed to poor compliance.

For more than two decades after the Centre for Disease Prevention and Control first, and subsequent documents were released, refined and mandated to be used for training and practice by all health personnel as a primary strategy to reduce the risk of transmission of micro-organisms, anecdotal observation in some units/wards of Federal Medical Centre Gombe showed poor/inconsistent hand washing, selective use of protective equipment such as gloves, eye goggles, apron and poor disposal of sharp consumables by health care providers. These practices posed high risks for the transmission of infections to the patients as well as the care providers. The resultant infection prolongs the patient's period of hospitalization, affects attention to family matters and additional financial burden which is sometimes difficult to meet up by many patients. The health workers are exposed to blood and other body fluids which lead to infections such as HIV and Hepatitis B following poor compliance to standard precaution hence the need to assess the knowledge and practice of standard precautions among nurses in Federal Medical Centre Gombe. 
1. To examine the level of compliance to the implementation of standard precautions among health care providers in FMCG.

2. To identify factors that affects the practice of standard precautions among health care providers in FMCG.

3. To determine the perception of nurses on how to improving the practice of standard precautions in FMCG.

\section{Methodology:-}

The methods used in research study is qualitative study. The research was completed using a comprehensive questionnaire. The questionnaire was filled through conducting structured and semi structured interviews, focus group discussions, and the data was triangulated by observations.

The quantitative data was analyzed using various data bases in excel, however; the concept of noticing, collecting and thinking is highly considered while analyzing the data for the research study.

The research study was conducted in governmental, semi- governmental and private hospitals. The researcher also conducted comparative qualitative analysis at the end of the work, where the current nursing practices and standards of the government hospitals was compared with semi - government and private ones.

\section{Study Design:-}

The research study design was considered to be qualitative observational and descriptive study. It is mentionable that this designed is selected just because the research did not intend to intervene, and it has also not considered any comparison group.

\section{Sampling Approach:-}

In order to properly implement this research, the researcher used one of the non-probability sampling methods, called purposive sampling. It is because the research is qualitative and mostly for qualitative researches one of the non-probability sampling methods is used. In purposive sampling the researcher defines some criteria's for the subjects and they are selected based on those criteria's.

\section{Inclusion/ Exclusion Criteria:-}

The researcher has considered the following exclusion and inclusion criteria to the conduct the research:

Inclusion Criteria's:

- Be a Kabul city based hospital.

- Provide secondary health services.

- Have OPD, IPD, surgical, and Medical wards. Be one of the government and private hospital.

clusion Criteria's:

- Hospitals outside Kabul city.

- Hospitals providing only primary health services such as BHC clinics.

- Small diagnostic clinics that does not have OPD, IPD, surgical and medical wards.

\section{Recruitment:-}

As part of the research, the data collection from 26 hospitals wasss done through trained data collectors. Initially the researcher did not plan to recruit someone for the data collection for two reasons; first the researcher didn't have any specific budget for this research, secondly the researcher wanted to ensure the quality of the data. Since the number of hospitals to be covered in Kabul reached to 26, and in one working day, it was not possible to cover more than one hospital, the researcher decided to collect data by some of the trained data enumerators. The enumerators were provided comprehensive training prior to data collection. It is mentionable that the cost for data enumerators was covered by the researcher himself.

\section{Data Collection and management:-}

The data was collected by researcher and by the trained hired data collector from the targeted hospitals. The data collection teams were consisted of male and female, the data from (Maternity hospitals) were collected through female data collectors and from other targeted hospitals, the data were collected by male data collectors. The females were hired just because of keeping the social norm and getting high quality data from the female respondents. 
In addition to that, various methods were used to collect good and sufficient data from the target respondents, these included but not limited to, detailed questionnaires, focus group discussions, and observation. The quality of the data was verified using liked skills (Triangulation) and it was ensured that the data collected is valid and reliable, the data was also checked and compared with the HMIS data of Public Health ministry and Public Health Directorate for capital hospitals.

The collected data was thoroughly entered to the excel data-base and following methods were used in each stage to produce proper analyses:

1. Pre-Analysis stage: in this stage the data was processed through editing, coding and feeding to the computer. In the second stage the data was tabulated as (mono, bi and multivariate) and then the data was distributed as frequency distribution, percentage distribution and mix methods of both types of distribution.

2. During Analysis: Data interpretation was done through following methods: Summarizing ( Based on similarities and differences) (people interviewed- (Male,female)-based on Gender similarities), Categorizing (Location, Age, etc).

3. Post Analysis: in this stage Proof Reading of the collected and analyzed data was done properly and was shared with the decision Makers.

\section{Research Tools:-}

The research was completed using a comprehensive questionnaire. The questionnaire was filled by conducting structured and semi structured interviews, focus group discussions and the data was triangulated by observations and verified with HMIS data of the Public Health Ministry.

\section{Pilot Testing:-}

The questionnaire was piloted in one of the hospitals (Ata-Turk Pediatric Hospital) and it was refined after the pilot testing. Later on the adjusted version of the questionnaire was utilized by the researcher in the roll out phase of the research.

\section{Ethics:-}

All the research ethics were considered during the research; because the provided data was not harmful for any person. Since this research was completely aimed for the public and government's good. Following research ethics were strongly followed.

- $\quad$ Not disclosing the data of one hospital to another.

- $\quad$ Being completely impartial in the whole research process.

- Get permission from the hospital managers before getting any type of pictures from the hospital.

\section{Research Outcome:-}

The research was completed considering the current nursing standards and at the same the researcher found the major causes as why the standard nursing practices are not followed in the targeted hospitals. Thus the research also provided valid recommendations in order to bring considerable changes in current practices and improve the quality of nursing services in the mentioned hospitals.

\section{Monitoring and data verification:-}

The researcher also ensured the quality of the data collected through data collectors, this was done through the following activities:

- $\quad$ Preparing and implementing the sample based plan.

- $\quad$ Avoiding sampling, and other types of biases.

- $\quad$ Collected data verification in case missing data is noticed.

- Piloting the questionnaire, before the roll- out phase of the research and refining and finalizing the questionnaire.

\section{Results:-}

This section of the research study is the main part or the analysis part of the research, in this section all the research data has been analyzed using various methods of qualitative and quantitative data analysis processes and tools. In this section actual personnel of the hospitals have been compared with UN- WHO standards for the purpose to find out as if the current number of nursing staff are according to the standards for the hospitals or not. In addition to 
that, nursing management system of governmental hospitals have been compared with public private partnership and private hospitals.

Moreover; the study has also covered the satisfaction level of nursing management with regards to current standards, knowledge about standard guidelines, availability of written standard nursing guideline in the hospitals, time management and initiatives taken by public private partnership and private hospitals have been considered.

\section{Doctor bed ratio and Nurse bed ratio:-}

One of the major complaints of the hospital managers was lack of staff in their relevant hospitals. In order to find out the seriousness and depth of this problem, one section of the research questionnaire asked about this problem from the respondents. The facts and figures collected in this research were than compared with WHO standards and conclusion was made accordingly.

Based on the UNWHO standards in the ward for four beds there has to be one doctors, and for two beds there have to be one nurse. However, in emergency section of the hospitals, for two beds there has to be one doctor.

The research data shows that all the hospitals have the required number of doctors and nurses, where the average doctor bed ratio in the governmental hospitals reached to 1.1 , which is high than the normal ratio of 0.25 , that means for each bed in the governmental hospitals they had a doctor and the average doctor bed ration in PPPs and private hospitals reached to 0.8 accordingly. Interestingly the average nurse bed ratio in all governmental, PPPs and private hospitals was found to be 0.5 , which is normal based on the WHO standards. For more details on hospital level please refer to the facts and figures in the hospital profile at the annex section, and for diagrammatic presentation of the facts and figures, please refer to figur1.

Table 1: Bed doctors and bed nurse ratios in governmental, PPPs and private hospitals

\begin{tabular}{|l|l|l|}
\hline & Average doctor Bed Ratio & Average Nurse Bed Ratio \\
\hline Government hospitals & 1.1 & 0.5 \\
\hline Private hospitals & 0.8 & 0.5 \\
\hline Public Private Partnerships & & \\
\cline { 2 - 3 } & 0.8 & 0.5 \\
\hline
\end{tabular}

The case is evident in the below table, that shows the overall average for bed nurse and bed doctors' ratio, compared to the UNWHO standards. As a result, it can be easily concluded that the number of doctors, and nurses are not less than the UNWHO standards.

Table 2:- Average bed nurse and bed doctor's ratio compared to WHO standards.

\begin{tabular}{|l|l|l|l|l|}
\hline Doctor & bed & Nurse Bed Ratio & Average doctor bed & Average nurse Bed Ratio \\
\hline \multirow{2}{*}{ Ratio } & \multicolumn{3}{|c|}{ Ratio } & 0.5 \\
\cline { 2 - 5 } & \multicolumn{3}{|l|}{0.9} & 0.5 \\
\hline
\end{tabular}




\section{Gender Analysis in nursing department's heads:-}

The research data shows that, most of the interviewed head of nurses were male nurses and only three female nurses were interviewed in maternity hospitals, and no female head nurse were interviewed other than maternity hospitals. It means that $92 \%$ of the head of nurses recruited in the targeted hospitals are male and only $8 \%$ of them are female. This means the number of the female nurses are very low, and in most of the hospitals such as pediatrics and maternity hospitals they are highly need female nurses to be deployed. Secondly the male nurses were currently involved in decision making and decreased number of female nurses couldn't influence to make their own decision for improvement of the nursing services. Thus the ratio of female nurses should be increased for those hospital where it is highly required. For graphic presentation of gender ratio, please refer to the below facts and figure.

\section{Standards of $\mathrm{WHO}$}

Actual deployment of the nurses and doctors

Table 3:- Gender analysis in nursing department heads.

Gender Percentage In Interviewed Group Of Nurses

\begin{tabular}{|l|l|}
\hline \multicolumn{1}{|c|}{ MALE } & \multicolumn{1}{c|}{ Female } \\
\hline 24 & 2 \\
\hline $92 \%$ & $8 \%$ \\
\hline
\end{tabular}

\section{Nurses Education level:-}

The research data also shows that, $80 \%$ of the interviewed nurses were graduated from the 15 grade from various institutes in Afghanistan. And 12\% of the nurses were graduated from 14 grade and $4 \%$ of them were graduated of 12 grade, and $4 \%$ of them had master degree in nursing care. This means most of the recruited nurses in governmental and PPPs and private hospitals are graduate nurses and only limited number of them are not graduated of nursing institute.

As looking to the overall graduation trend, $16 \%$ of the interviewed nurses were having low level of education. And this low level of their education has caused little contribution to the improvement in nursing services or following the nursing standards in the hospital.

Table 4:- The education level of the recruited nurses in the targeted hospitals. NURSES EDUCATION LEVEL

\begin{tabular}{|l|l|l|l|l|}
\hline $\begin{array}{l}\text { GRADUATE OF 12 } \\
\text { GRADE }\end{array}$ & \multicolumn{1}{|c|}{ Graduate of 14 Grade } & \multicolumn{1}{c|}{ Graduate of 15 grade } \\
\hline 1 & 3 & 21 & 1 \\
\hline $4 \%$ & $12 \%$ & $80 \%$ & $4 \%$ \\
\hline
\end{tabular}

Trainings Conducted to the Nursing staff:-

The research data shows that $65 \%$ of nursing department heads have received trainings from MOPH (Ministry of Public Health) and had knowledge of standard nursing precautions and standard care, and $35 \%$ have not received training from MOPH. In addition to that, all nurses in PPPs which makes $8 \%$ of the whole target group have received $100 \%$ full training package and they were enrolled in regular cycle of nursing trainings from their hospital through an organized capacity building programs. The data also revealed that $43 \%$ nurses working in private hospital have also received various nursing practices trainings from private hospitals and $57 \%$ of the nurses in the target private hospital didn't received trainings on standard nursing care. Looking to the above facts $g$ that most of the nurses have received nursing trainings, but still the quality of delivering the nursing services was poor. And main caused found for this was that the nurses in all MOPH related hospital have received training in only one or two topics of the standard nursing packages. Thus if they would have received all and full package of training on nursing standards, they would have obliviously delivered standard nursing care to the patients. In addition to that it was also found that the nurses are not provided the required infection prevention materials, which is another reasons that if they aren't given infection prevention and other materials that are regularly used to protect patient and nurse, they wouldn't be able to reach the goal which is universal precaution for patient and nurse. 
Table 5:- Shows the trainings conducted to the nursing staff in hospitals. Training On Nursing Standards

\begin{tabular}{|l|l|l|l|}
\hline & Description & Frequency & Percentage \\
\hline GOVERNMENTAL MOPH & Training provided & 11 & $65 \%$ \\
\hline & Training not provided & 6 & $35 \%$ \\
\hline PPPS & Training provided & 2 & $100 \%$ \\
\hline & Training not provided & & $0 \%$ \\
\hline PRIVATE & Training provided & 3 & $43 \%$ \\
\hline & Training not provided & 4 & $57 \%$ \\
\hline
\end{tabular}

In addition to that the researcher also found that the head nurses are not cascading the received trainings to the subordinates as well. The trainings provided to the nurses in governmental hospitals are mentioned: Patient safety, nursing standards, IP, Center supply IP, IV medicine injection IP, IV medicine injection application, HMIS, waste management, Caring psychiatric patients, IV injection, psychiatric diseases, IP, waste management, external and environmental prevention of hospital, Caring psychiatric patients, IV application, psychiatric diseases, IP, waste management, external and environmental prevention of hospital, IP, waste management, external and environmental prevention of hospital, IP, IV medicine injection application, Besides that, it is also noticed that the PPPs are the only hospitals that have comprehensive continuous nursing capacity building plan and training programs. The PPP are conducting full package of the training to the nursing staff.

The trainings provided to the nurses in the PPPs are:

- $\quad$ Basic life support

- Advance life support

- $\quad$ dressing and IV drug application

- $\quad$ Nursing standards \& universal precautions

- Neonatal care

- Maternity and child health

- Infection prevention

- Waste management

The researcher also found that some of the private hospitals also had nursing training programs to build the capacity of their nursing staff, but those trainings were also covering limited topics and were for limited duration as well.

The trainings provided to the nursing staff of the government, is not enough and those were also not cascaded to their subordinates. And regular capacity building plan were also nor existing in each governmental hospital.

The nursing staff working in the MOHE (Mistry of higher education) related hospitals, have not received one training, this has additionally affected the delivery of standard nursing services and practicing based on the standards. The causes that why they weren't not provided trainings were found that the MOHE was lacking the budget, and they was also no guideline given to their nursing staff. This also shows that the coordination between the ministries were very weak, if it would have coordinated the developed guideline from MOPH could have distributed to the nursing staff of the MOHE from MOPH without any further addition of resources for newly development of the nursing standards guidelines. It was also found that most of the private hospitals also don't have any plan for capacity building of the nursing staff and aren't providing training to build the capacity of their nursing staff.

\section{Written nursing standard guideline:-}

The data shows that some the governmental hospitals related to the MOPH have received the standard nursing guideline and they knew that the MOPH have developed these guidelines for nursing care during delivering of nursing services to the patients.

The data shows that, $62 \%$ of the hospitals did not receive the standard guidelines from the MOPH, and only $38 \%$ of the hospitals have received the standard guidelines from the MOPH.

The most interesting fact was that the hospital had received only one copy of the standard guideline document, however; that copy are being kept in the shelves, and haven't been in use to in line their nursing services according 
to the guidelines. No follow-up for the set standards are ever conducted by the hospital management or the MOPH in more than $90 \%$ of the hospital. The remaining $10 \%$ of the hospital management just check nursing practices through limited indicators. However; the documented data for improvement of the nursing services or the action points of the controlling committees were also not existing in the targeted hospitals.

Table 6:- Hospitals received and not received nursing guidelines. Nursing Standard Guidelines

\begin{tabular}{|l|l|l|}
\hline \multicolumn{1}{|c|}{ HOSPITALS NOT RECEIVED } & \multicolumn{1}{|c|}{ Hospitals received } & \\
\hline 16 & 10 & \\
\hline $62 \%$ & $38 \%$ & \\
\hline
\end{tabular}

The data also shows that among 10 of the hospitals who received the guideline, $80 \%$ of the hospital nursing staff didn't know, whether the developed guideline are updated since its first pollution or not. And only $20 \%$ of them had the information that the guidelines are once update after its first pollution.

Table 7:- The awareness of nurses on the last updated version of the nursing guidelines.

\begin{tabular}{|l|l|l|}
\hline & \multicolumn{1}{|c|}{ IS THE GUIDELINE UPDATE? } \\
\hline & \multicolumn{1}{c|}{ Yes it is updated } \\
\hline 8 & 2 & 2 \\
\hline $80 \%$ & & $20 \%$ \\
\hline
\end{tabular}

The researcher also inspected the issue of development of the guidelines from the hospitals who have received them, it was found that $66 \%$ of the 10 hospitals who have received the guidelines said that, this text guide for nursing practices is developed by the MOPH, however $11 \%$ of the hospital nurses said that the guideline that they received is developed by the MOPH and LMG and only $23 \%$ know that the guidelines are developed by the MOPH and USAID. It means that most of the nursing staff didn't know about that whether the developed guidelines are from MOPH or there is any contribution of any other organization or not.

Table 8: The awareness of nursing staff on whom have developed the nursing guideline. IS THE NURSING GUIDELINE DEVELOPED BY MOPH OR ANY OTHER AGENCY

\begin{tabular}{|l|l|l|}
\hline \multicolumn{1}{|c|}{ BY MOPH } & \multicolumn{1}{|c|}{ BY MOPH and LMG } & \multicolumn{1}{|c|}{ BY MOPH \& USAID } \\
\hline 6 & & \\
\hline $66 \%$ & 1 & 2 \\
\hline
\end{tabular}

The research data also shows that the 10 of the hospital who have received the guidelines, $90 \%$ of the nursing department heads mentioned the following topic as included in the text guidelines:

- IP or infection prevention

- $\quad$ IV medication guideline

- $\quad$ patient and nurse safety

In addition to that, only $10 \%$ of the nursing department head said that the topic that are included in the guideline are as below:

- $\quad$ Standards are for OPD,

- Recovery,

- Maternity ward,

- Rehabilitation department,

- Operation theater,

- Neonate section and so on.

However; considering the above topics, actually the guideline includes the following main topics as nursing standards and nursing guideline:

- Patient checkup

- Infection control, safety and prevention

- Patient hygiene and comfort 
- $\quad$ Patients IV Medicine application and documentation

- Dead body care

- Insertion of tubes

- IV medicine injections

- Dressing and

- Patient care

- $\quad$ Nursing management

In view of the above discussions, it can be confidently stated that more than $90 \%$ of the nurses in the mentioned governmental hospitals didn't know even about the topics of the nursing standards and guidelines. However; it is mentionable that they even didn't know, what topics are part of the standard guideline, therefore; they aren't able to practice nursing standards in delivery of the nursing care to the patients.

\section{Knowledge on nursing standards:-}

The research data shows that the interviewed nurses didn't have knowledge on nursing standard, precaution methods and procedures, and most interestingly they didn't know on how to follow the standard precaution practices during the delivery of nursing services to the patients. However; the research also shows that most of such cases were visible in the government hospitals. And the reason for delivering of the poor quality nursing services from the government hospitals was lack of resources for the nursing staff in the hospital. And low level of knowledge of the nursing staff on nursing standards. It was also found that some of the hospital such as FMIC, CURE hospitals, was delivering high quality nursing services to their patients. The reason for doing so, was the availability of nursing required standards, resources, regular capacity building programs, timely feedback of the hospital management and receiving client feedback were the main reason for their high and quality nursing services.

Table 9: Shows knowledge level of nursing staff on nursing standards.

\begin{tabular}{|l|l|}
\hline $\begin{array}{l}\text { HAVE KNOWLEDGE ON } \\
\text { NURSING }\end{array}$ & \multicolumn{1}{|c|}{ Does not have knowledge on Nursing standards } \\
\hline STANDARDS & \\
\hline 11 & 15 \\
\hline $19 \%$ & $81 \%$ \\
\hline
\end{tabular}

\section{Monitoring of the nursing services:-}

The data shows that the health services being delivered by the hospital is monitored. $76 \%$ of them were monitored by the MOPH and most of the hospital monitored by the MOHP were the governmental hospital. However; 24\% of the hospital replied as their health facilities and health services of aren't monitored by MOPH. And most of these hospitals are the private health facilities which aren't monitored by the MOPH. The research data also shows that the monitoring visits are mostly conducted on monthly basis to each health facility, and the main observation from the monitoring is focused on the availability of the staff in the hospital not the quality or delivery of the nursing services that are delivered from the hospital.

Table 10: Shows external monitoring of the nursing practices through MOPH.

\begin{tabular}{|l|l|}
\hline \multicolumn{1}{|c|}{ YES MONITORED } & \multicolumn{1}{c|}{ Not Monitored } \\
\hline 20 & 6 \\
\hline $76 \%$ & $24 \%$ \\
\hline
\end{tabular}

\section{Satisfaction level:-}

The data shows that $12 \%$ of the research coverage hospital were highly satisfied of their nursing services delivered to the patients, and $50 \%$ of them were satisfied only from the nursing practices and standards that are following during the delivery of health services. In addition to that only $38 \%$ of the hospital management were not satisfied from the nursing practices in their hospital of various reasons, and wanted an major change in the process, equipment's and materials to deliver quality health services to the patients. The reasons for satisfaction and dissatisfaction is mentioned in table 5. 
Table 11:- The satisfaction level of hospital management from their nursing services.

\begin{tabular}{|l|l|l|}
\hline \multicolumn{1}{|c|}{ HIGHLY SATISFIED } & \multicolumn{1}{|c|}{ Satisfied } & Not Satisfied \\
\hline 3 & 13 & 10 \\
\hline $12 \%$ & & \\
\hline & $50 \%$ & $38 \%$ \\
\hline
\end{tabular}

\section{Discussions:-}

\section{Research Coverage:-}

The research was done in Kabul based 26 tertiary health service delivery hospitals, including governmental, public private partnership hospitals and private hospitals. Out of 26 hospitals 17 of them were governmental hospitals, including 14 managed by ministry of public health and 3 managed by ministry of higher education and two of them were public private partnership, ad seven of them were private hospitals. For more details, please refer to the annex.

\section{Major Constraints:-}

The research itself is a challenging task, however in Afghanistan the current bureaucratic system further makes it challenging. Here getting accurate data from any institution is a challenge. In order to discuss the main constraints faced by the researcher, please refer to table 4 .

Table 4: Main constraints faced by the researcher during the research

\begin{tabular}{|l|l|}
\hline S.N & \multicolumn{1}{c|}{ Major Constraints } \\
\hline $\mathbf{1}$ & Due week coordination mechanism between MOPH and PPPs the collection of data was delayed. \\
\hline $\mathbf{2}$ & The hospital directors in many cases were not cooperative at all, they did not provide us the data. \\
\hline $\mathbf{3}$ & Lack of budget was also one of the constraints, because the research was completely funded by the \\
\hline & researcher himself, and the research was designed in such a way that the data collector was supposed to \\
\hline & visit all 26 Kabul based hospitals. \\
\hline $\mathbf{4}$ & $\begin{array}{l}\text { Leaving the hospital early than the official hours caused significant delays in data collection of the } \\
\text { research. }\end{array}$ \\
\hline $\mathbf{5}$ & Unavailability of previous researches in the quality aspect of the health care service delivery in Afghanistan \\
\hline & was also a challenge. \\
\hline $\mathbf{6}$ & The MOPH website was not updated with uploading the policies and strategies of the ministry, thus need \\
\hline & to take in person from its relevant department. \\
\hline
\end{tabular}

Key Results of the research:-

Doctor bed ratio and Nurse bed ratio: The target hospitals have the required number of doctors and nurses, where the average doctor bed ratio in the governmental hospitals reached to 1.1, which is high than the normal ratio of 0.25 . Interestingly the average nurse bed ratio in all governmental, PPPs and private hospitals was found to be 0.5 , which is normal based on the WHO standards.

Trainings Conducted to the Nursing staff: $65 \%$ of nursing department heads have received trainings from MOPH, $35 \%$ did not. In addition to that, all nurses in PPPs have received 100\% full training package. $43 \%$ nurses were trained on nursing standards and $57 \%$ still did not received training on nursing standards.

Written nursing standard guideline: $62 \%$ of the hospitals did not receive the standard guidelines from the MOPH, and only $38 \%$ of the hospitals have received the standard guidelines from the MOPH. The PPPs had $100 \%$ their nursing guidelines.

Knowledge on nursing standards: 19\% of the interviewed target group had awareness on nursing standards and $81 \%$ of the target group (MOPH \& private hospitals) did not have awareness and knowledge on nursing standards. However; PPPs nurses were 100\% aware of the nursing standards and nursing guidelines.

Monitoring of the nursing services: $76 \%$ of the hospitals were monitored by the MOPH and most of the hospital monitored by the MOHP were the governmental hospital. However; $24 \%$ of the hospital replied as their health facilities and health services of aren't monitored by MOPH. 


\section{Challenges implementing the nursing standards:-}

Following were the challenges found to be the main constraints to implement the nursing standards in the target Kabul based hospitals:

1. Lack of proper knowledge on the concept or on how to implement the process has been found to be the most important factor in preventing a proper implementation of the process.

2. Despite awareness of the need for registration of nursing process, nursing records were not complete, this needs attitude change in nursing behaviors.

3. Required materials for nursing care and maintaining the nursing standards were not provided to the most of the hospitals by its line ministry.

4. High cost of nursing standards and free of charge health services, reduce the subsidization capacity of government to timely provide required nursing care materials to implement the standard nursing processes.

5. Lack of enough time for the process due to large number of patients can cause an impediment to perform the nursing process.

\section{Main comparisons:-}

a. The head nurses whom received training from MOPH was not a full package of the nursing standards. It hardly included only two or maximum three topics of the whole package.

b. At the second stage the nursing heads were not being followed to cascade the training to its sub-ordinates.

c. The training provided to the nursing department heads were only one in most of the cases throughout the year with covering minimum topics of the package.

d. Access to the nursing guidelines was limited to only one copy per hospital.

e. The situation comparing to the PPPs, it was found, that the PPPs were providing regular trainings to its nursing staff.

f. The PPPs, provided full package nursing standards training to the nursing staff, as well as, they had given access to each nurse through a software equipment's to the guidelines.

g. Required nursing care materials were provided to the nursing staff by PPPs on time, and with required quantity.

h. Exposure visits and regular on the job, off the job, and abroad trainings were provided to the PPPs nursing personnel.

i. Regular management follow-up to implement the standard nursing processes were found to be the strength point of the PPPs, however; the MOPH and MOHE were not even monitoring their nursing staff for nursing procedures and implementation of nursing care guidelines.

\section{Generalizability of the research:-}

The research was made in very short time facing many challenges, thus further researches are recommended to understand the knowledge level of the nurses, impact of the nursing training in service delivery, load of patient in service delivery, provision of nursing care material in meeting standards, nursing practices against standards etc.

The result achieved by the research can be further explored and take benefit for future researches, but can't be generalized for all hospitals.

\section{Recommendations:-}

This section includes recommendations for improvement of overall nursing services in target hospitals. This section also gives strategic inputs for the policy makers to add some of the crucial points in the updated version of MOPH policies.

Regular training on full package of nursing guidelines should be provided to the nursing staff to build their capacity.

Timely follow up of nursing management with the trained nurses on implementing the standard nursing processes. This may need time but will certainly bring attitude changes in the nursing staff.

Required infection prevention materials and medicine for emergency patient should be given in enough quantity and on timely manner to the nursing care departments.

Access to the nursing guideline should be given to all hospitals through uploading in the MOPH website.

Monitoring and feedback mechanism should be in place for nursing practices and nursing care should be monitored 
on regular basis from the $\mathrm{MOPH}$.

MOPH should introduce user fee to each patient to reduce the cost of subsidization and in the meantime support hospital with provision of the required nursing care materials.

Exchange learning programs from government to PPPs and peer learning should be introduced to build the capacity of the government hospital nursing staff.

Annexes:-

Annex1. Shows the questionnaire used for data collection.

\section{SECTION NO.1: IDENTIFICATION:}

\subsection{Date?}

1.2 Name of focus group discussions participants:

1.3 Position:

1.4 Education level? a) Secondary school b) graduate c) Diploma d) others

$\begin{array}{llll}1.5 & \text { Sex? } & \text { A) Male } & \text { B) Female }\end{array}$

1.6 \# of years of nursing experience? a) 2-3 b) 3-5 c)5-10 $\quad$ d) More than 10 years

1.7 Have you ever received any standard precaution knowledge nursing training? a) Yes b) No

1.7.1 If Yes, Please tell us the name of the training? (

1.7.2 Which entity conducted the training (

1.7.3 Duration of the training? (

1.7.4 Main topics covered under the training? a)

)

)

)

b)

\section{Section 2: Current standard nursing practices of the hospital?}

2.1 Does the hospital have any written health standard precaution guideline or mechanism for nursing?

2.1.1 If yes, when was the standard precaution guide line developed?

2.1.2 When the standard precaution guide line was last updated?

3.1.1 Has the system been developed for you by Ministry of public health? a) Yes $\quad$ b) No

2.1.3 If yes, which organization has developed it for? (

2.1.4 If yes, what are the different components of it?

2.1.5 Are you satisfied with current nursing process followed in your hospital? a) Yes b) No

2.1.6 If yes, what is your satisfaction level a) highly satisfied b) satisfied c) not satisfied

2.1.7 Reason for your satisfaction?

2.1.8 If not, what are the reasons?

2.1.9 How do you ensure that standards are maintained by nurses during caring patients?

2.1.10 Do you have any measurement for maintaining the level of standards? If yes please explain. If no: what are the causes?

2.1.11 What changes would you recommend for improvement of standard precautions process here?

2.1.12 Has some one ever monitored the standards of nursing care of health service delivery in your hospital? a) yes b) no

2.1.13 If yes which organization (

2.1.14 How often does the monitoring happen a) weekly b)monthly $\quad$ c) Quarterly d) Annually

2.1.15 General Observation and triangulation?

\section{SECTION NO.3: KNOWLEDGE ABOUT STANDARD PRECAUTIONS:}

3.1 Do you know what standard precaution is?
a) Yes
b) $\mathrm{No}$
c) Uncertain

3.2 Standard precaution is only applicable for the patients with the confirm diagnosis of infection or in latent period of infection:
a) Yes
b) No
c) Unknown

3.3 The main goal to implement standard precaution is to protect the medical staff: 


\section{$\begin{array}{lll}\text { a) Yes } & \text { b) No } & \text { c) Unknown }\end{array}$}

3.4 Washing and disinfecting the hands immediately if contacting any blood, body fluid, secretion, excretion, or dirty substance:
a) Yes
b) No
c) Unknown

3.5 Washing the hands if contacting different patients:
a) Yes
b) No
c) Unknown

3.6 Since the gloves can prevent from the pollution for the hands, there is no need to wash hands after taking off the gloves:
a) Yes
b) $\mathrm{No}$
c) Unknown

3.7 It shall be avoided for the polluted protective articles to contact with the surface of other articles, the clothes or the staff outside of the ward:
a) Yes
b) No
c) Unknown

3.8 It shall not be shared for the personal protective articles, such as the gloves, the mask, etc:
a) Yes
b) No
c) Unknown

3.9 The gloves cannot be worn in the oral nursing operation, which may contact the mucosa of patient:
a) Yes
b) $\mathrm{No}$
c) Unknown

3.10 The gloves shall be wore in the operation might contact with the section and excretion of patient:
a) Yes
b) No
c) Unknown

3.11 The gloves should be worn in the operation of blood drawing, venous puncture, etc:
a) Yes
b) $\mathrm{No}$
c) Unknown

3.12 The gloves shall be changed if contacting different patients:
a) Yes
b) No
c) Unknown

3.13 The face mask or mask shall be worn in the operation might induce the spraying of blood, body fluid, secretion or excretion:
a) Yes
b) $\mathrm{No}$
c) Unknown

3.14 The protective eye patch or goggle shall be worn in the operation might induce the spraying of blood, body fluid, secretion or excretion:
a) Yes
b) $\mathrm{No}$
c) Unknown

3.15 The protective suit shall be worn in the operation might induce the spraying of blood, body fluid, secretion or excretion:
a) Yes
b) No
c) Unknown

3.16 The protective cap or shoe shall be worn in the operation might induce the spraying of blood, body fluid, secretion or excretion to pollute the hair or shoe:
a) Yes
b) No
c) Unknown

3.17 The sharp disposal box shall be put in the area close to the sharp applicable area, forbidden the returned operation of second-hand pinhead, as well as the transformative pinhead application.

If the pinhead must be returned, it shall be processed by single hand:
a) Yes
b) No
c) Unknown

3.18 The caring for patients with HCV or syphilis only needs the standard precaution:
a)Yes
b) No
c) Unknown

3.19 The caring for patients with active pulmonary tuberculosis or varicella needs the standard precaution associated with the prevention from the airborne diseases.
a) Yes
b) No
c) Unknown

3.20 The caring for patients with intestinal infection or skin infection needs the standard precaution associated with the prevention from the diseases by contact transmission.
a) Yes
b) No
c) Unknown

\section{SECTION NO.4: General Observation and triangulation?:}

4.1 Have gown while caring the patients. a) Yes

4.2 Have washed hands before wearing the gloves? a) Yes

4.3 Wear gloves while nursing or taking care of patients? a) Yes

4.4 Wear mask during taking care of patients? a) Yes

4.5 Wearing protective cap during taking care of patients? a) Yes

4.6 Have knowledge of sharp disposals? a) Yes

4.7 Have knowledge of waste disposal? a) Yes

b) No

b) $\mathrm{No}$

4.8 Have the special sharp disposal box in your department and caring wards? a) Yes b) $\mathrm{No}$

b) No

b) $\mathrm{No}$

b) No b) No 
4.9 Have washed hands after gloves removing? a) Yes $\quad$ b) No

4.10 Wearing protective eye patch and goggle to protect the eyes in the operation? a) Yes

b) No

Name of the data collector: Position:

Contact details: Signature:

Name of the data provider: Occupation:

Contact details: Signature:

Annex.2: shows the time line of the research completed. The research was completed in three months based on the following detailed schedule.

\begin{tabular}{|c|c|c|c|}
\hline & Activity & & Timeline \\
\hline & & From & To \\
\hline 1 & Finalizing the research problem & October 25 & October 30 \\
\hline 2 & Literature review & November 01 & November 05 \\
\hline 3 & Designing research tools, techniques and methodolog & November 05 & November 10 \\
\hline 4 & Piloting the research in one of the hospitals & November 10 & November 15 \\
\hline 5 & Refining the tools based on the recommendations. & November 10 & November 15 \\
\hline 6 & Selecting data collectors, especially for female hospita & November 15 & November 20 \\
\hline 7 & Training them & November 15 & November 20 \\
\hline 8 & Data collection & November 20 & December 20 \\
\hline 9 & Data verification & December 20 & December 30 \\
\hline 10 & Data entry, processing, editing, coding & January 02 & January 10 \\
\hline 11 & Data analysis & January 10 & January 30 \\
\hline 12 & Report Writing & January 30 & February 17 \\
\hline 13 & Presenting the main findings of the research & February 05 & February 20 \\
\hline
\end{tabular}

Annex.3: Shows the List of the target hospitals.

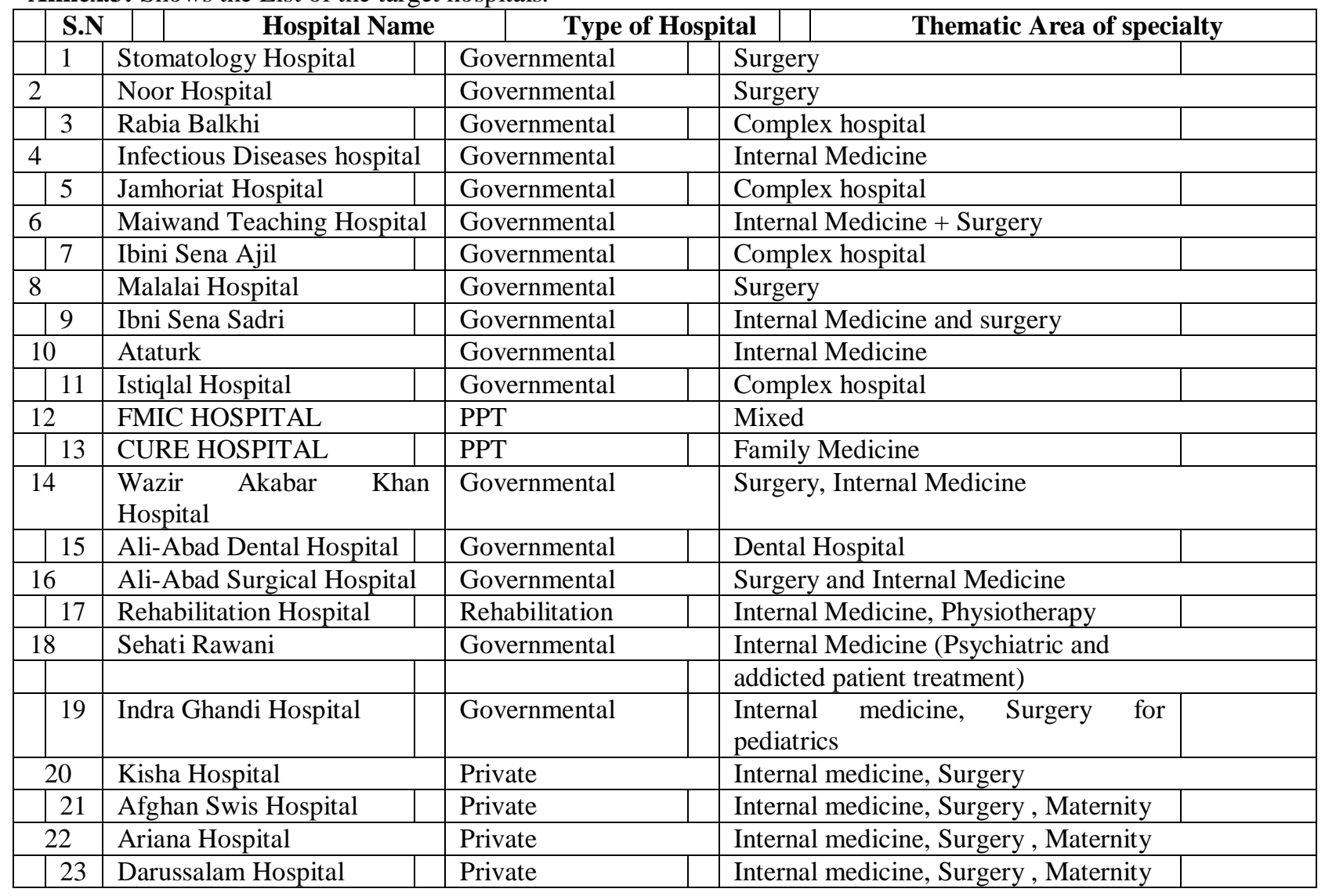




\begin{tabular}{|c|c|c|c|}
\hline 24 & Waris Wardak Hospital & Private & Internal medicine, Maternity \\
\hline 25 & Wardak Hospital & Private & Internal medicine, Maternity \\
\hline 26 & Spinghar Hospital & Private & Internal medicine, Maternity \\
\hline
\end{tabular}

Annex.4: Shows the List of references used for research.

\section{References:-}

1. Abubakar, S. M. (2014). Direct Res. J. Health Pharm. 2. Assessment of knowledge and practice of standard precautions among nurses working at Federal Medical Centre Gombe, Nigeria, 11.

2. Factors influencing nurses' compliance with Standard Precautions in order to avoid occupational exposure to microorganisms: A focus group study. (2011). BMC Nursing, 11.

3. Fayaz, S. H. (2012). Knowledge and practice of universal precautions among health care workers in four national hospitals in Kabul, Afghanistan. Kabul: J Infect Dev Ctries 2014; 4(8):535-542.

4. Health, M. o. (2011-2015). National Policy and Strategy for Nursing and Midwifery Services 2011 - 2015.

5. Kabul: MOPH.

6. Health, M. o. (2012). guideline on standarad nursing practices. Kabul: MOPH.

7. Luo, Y. (2009). Factors impacting compliance with standard precautions in nursing, China. Y. Luo et al. / International Journal of Infectious Diseases 14 (2010) e1106-e1114, 9.

8. Organization), W. (. (2004). Practical Guidelines for Infection Control in Health Care Facilities. Delhi: SEARO Regional Publication No. 41. 